\title{
Soft Perovskite-Type Antiferroelectric with Giant Electrocaloric Strength Near Room Temperature
}

\author{
Maofan Li, ${ }^{\ddagger}$, Shiguo Han, ${ }^{\ddagger}, \dagger$ Yi Liu, ${ }^{\ddagger, \dagger}$ Junhua Luo, ${ }^{\ddagger},, \dagger$ Maochun Hong, ${ }^{\ddagger} \S$ and Zhihua Sun ${ }^{*, \neq, \S, \dagger}$ \\ ‡ State Key Laboratory of Structural Chemistry, Fujian Institute of Research on the Structure of Matter, Chinese \\ Academy of Sciences, Fuzhou, Fujian 350002, China \\ $\S$ Fujian Science \& Technology Innovation Laboratory for Optoelectronic Information of China, Fuzhou, Fujian 350108, \\ China \\ † University of Chinese Academy of Sciences, Beijing, 100049, China
}

\section{Experimental Procedures}

Synthesis of (IA) ${ }_{2} \mathbf{C s P b}_{2} \mathrm{Br}_{7}(1)$ : Compound 1 was synthesized in the concentrated HBr solution $(40 \mathrm{ml})$, containing the stoichiometric ratio of $\mathrm{PbCO}_{3}(2.67 \mathrm{~g}, 0.01 \mathrm{~mol}),\left(\mathrm{CH}_{3}\right)_{2} \mathrm{CH}\left(\mathrm{CH}_{2}\right)_{2} \mathrm{NH}_{3} \mathrm{Br}$ $(1.68 \mathrm{~g}, 0.01 \mathrm{~mol})$ and $\mathrm{Cs}_{2} \mathrm{CO}_{3}(0.815 \mathrm{~g}, 0.0025 \mathrm{~mol})$. The bright yellow solution was initially formed with the boiling, which yielded the yellow powder precipitation after cooling down to room temperature. Subsequently, the saturated solutions of 1 were prepared at $\sim 363 \mathrm{~K}$ and maintained for 2 days, and bulk yellow crystals with size up to $18.5 \times 19.1 \times 1.8 \mathrm{~mm}^{3}$ were obtained by the temperature-cooling method with a cooling rate of $\sim 0.3 \mathrm{~K} /$ day. Powder X-ray diffraction data was measured on a Mini Flex 600 Powder X-ray diffractometer at room temperature, and the diffraction patterns were collected in the $2 \theta$ range of $5^{\circ}-45^{\circ}$ with a step size of $0.02^{\circ}$ (Figure S1).

Single crystal structure determination: Single crystal X-ray diffraction of $\mathbf{1}$ was obtained on a D8 diffractometer using the Mo K $\alpha$ radiation at $310 \mathrm{~K}, 323 \mathrm{~K}$ and $373 \mathrm{~K}$. Crystal structures of 1 were solved by direct methods and then refined by the full-matrix least-squares refinements on $F^{2}$ using SHELXLTL 97 software package. All the non-hydrogen atoms were refined anisotropically based on all reflections with $I>2 \sigma(I)$, and the $\mathrm{H}$ atoms were generated by geometrical considerations and placed at their idealized positions. Crystallographic data and structure refinements for 1 are given in Table S1. CCDC 2013896, 2013897 and 2013906 contain the crystallographic data in this paper. 
PXRD patterns were collected by using the MiniFlex 600 X-ray diffractometer at room temperature. Differential scanning calorimetry (DSC) measurement was performed on the NETZSCH DSC $200 \mathrm{~F} 3$ with the heating/cooling rates of $10 \mathrm{~K} / \mathrm{min}$. During the dielectric, pyroelectric and ferroelectric measurements, thin single-crystal plate samples with the thickness of $\sim 0.8 \mathrm{~mm}$ were cut. The polar crystallographic $c$-axis direction was verified by the X-ray diffraction. The dielectric constants $\left(\varepsilon^{\prime}\right)$ were measured using the two-probe AC impedance method with an Impedance Analyzer (TH2828A). The measurement of pyroelectric current was performed using an electrometer (Keithley 6517B) under the zero-electric field in the heating mode at the rate of $10 \mathrm{~K} / \mathrm{min}$. Moreover, the electric polarization versus electric field $(P-E)$ hysteresis loops of $\mathbf{1}$ were measured using the single-wave and double-wave method at different temperatures. The crystal samples of $\mathbf{1}$ with the polished surface were used to measure the variable-temperature SHG properties. The tunable laser $(\lambda=1340 \mathrm{~nm}$, the pulse duration is $1 \mathrm{~ns}$ and peak power is $\sim 2.0 \mathrm{MW}$ ) was employed as the fundamental light source, and SHG signals were collected using a Fluorescence Spectrometer (FLS 920, Edinburgh Instruments) at different temperatures. 


\section{Figures}

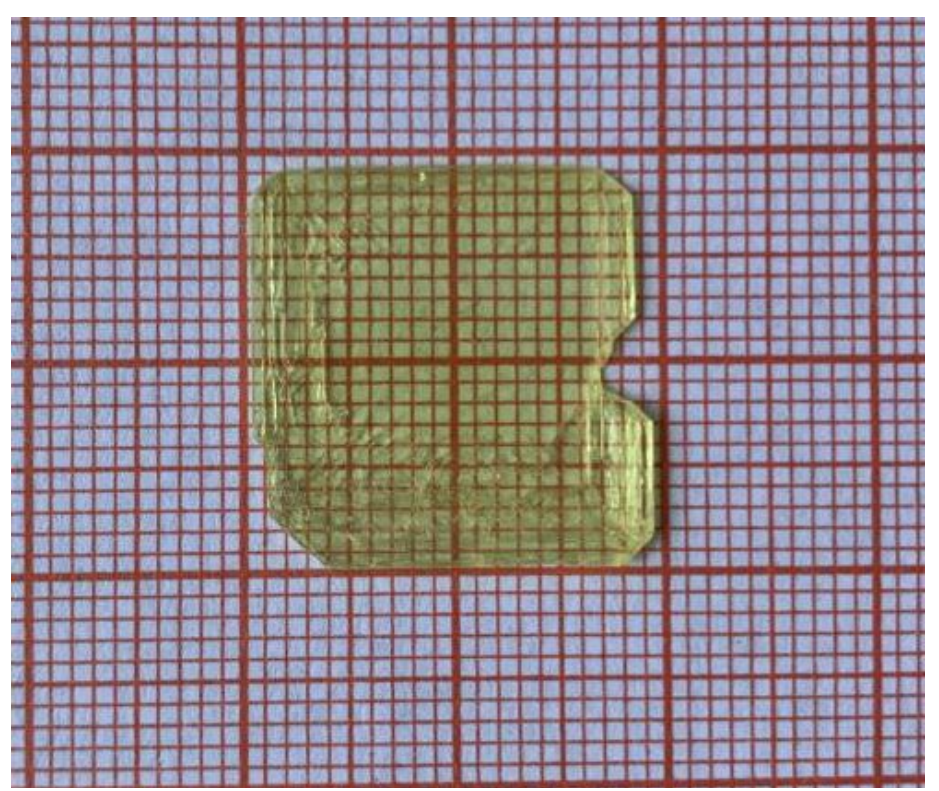

Figure S1. Large-size crystal of 1 with the size of $19 \times 20 \times 1.8 \mathrm{~mm}^{3}$

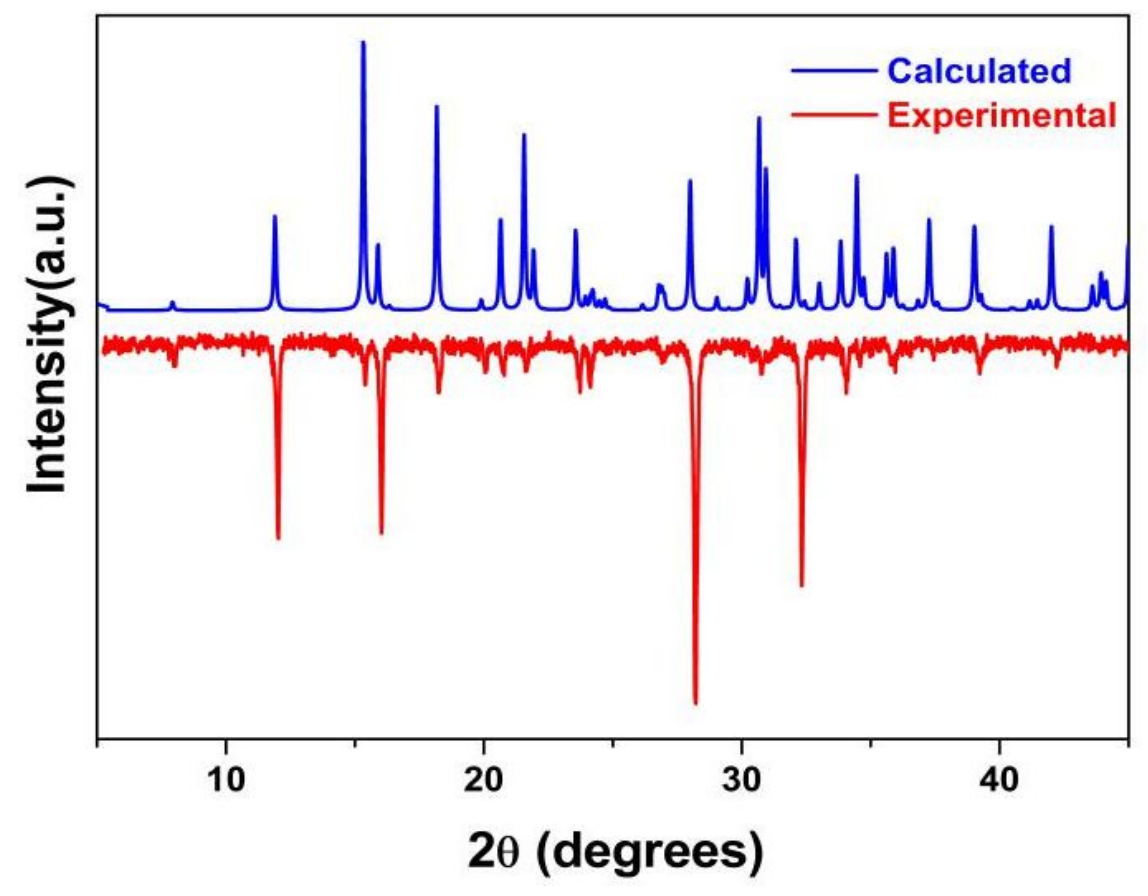

Figure S2. Experimental and calculated PXRD patterns for $\mathbf{1}$. 


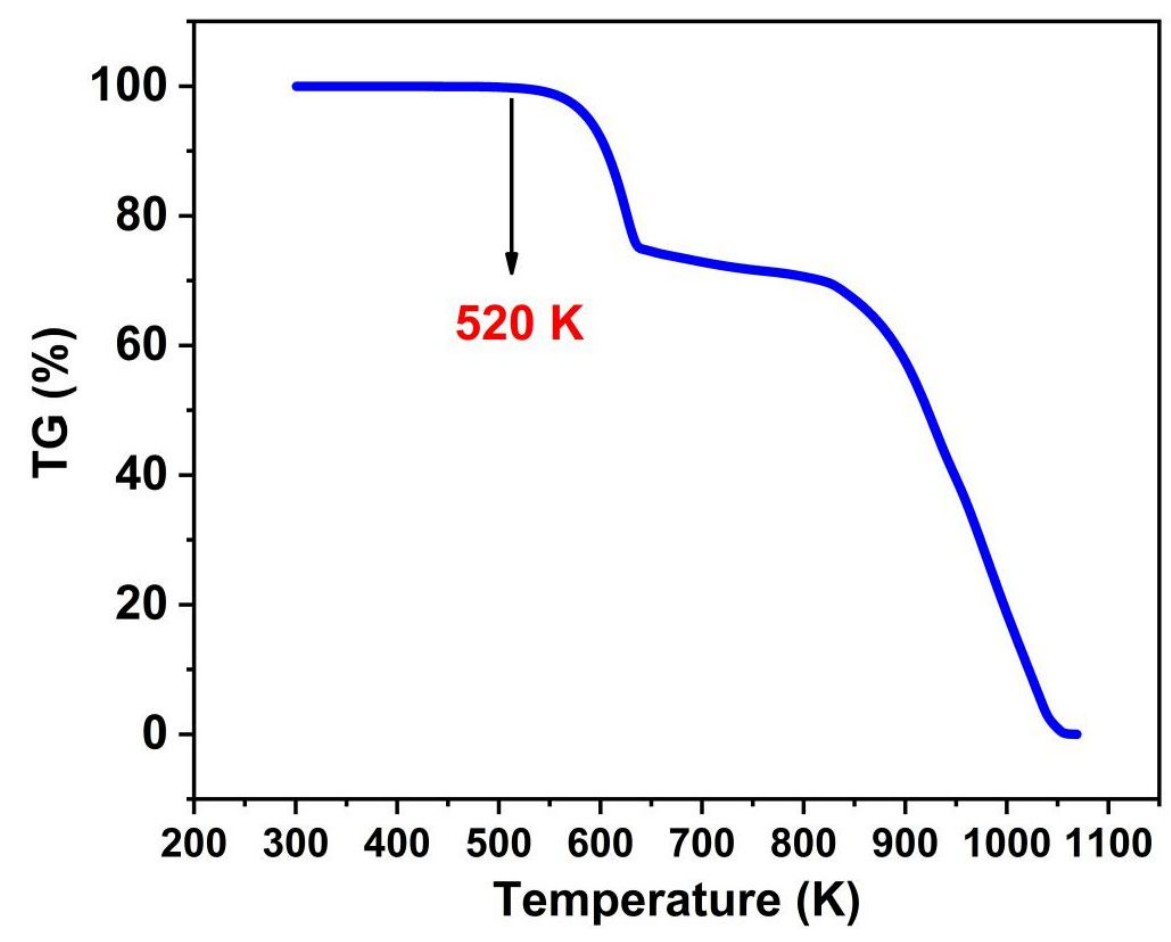

Figure S3. Thermogravimetric trace for 1, revealing its thermal stability up to $\sim 520 \mathrm{~K}$.

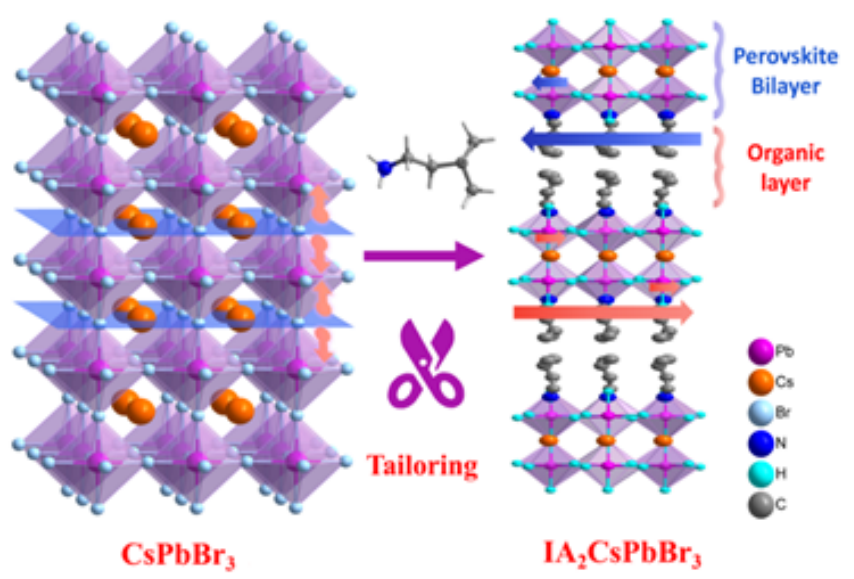

Figure S4. Scheme for the design of 2D architecture of 1 by alloying cation into 3D $\mathrm{CsPbBr}_{3}$-based motif. Basic structure for 1 consists of $\left[\mathrm{Pb}_{2} \mathrm{Br}_{7}\right]_{\infty}$ networks and organic $\mathrm{IA}^{+}$spacer, and the $\mathrm{Cs}^{+}$ cation locates inside the cavity formed by $\mathrm{PbBr}_{6}, \mathrm{Octahedra}$ Two sheets of interlayer organic $\mathrm{IA}^{+}$ cations are linked to inorganic perovskite bilayers through $\mathrm{N}-\mathrm{H} \cdots$ Br hydrogen bonds. 


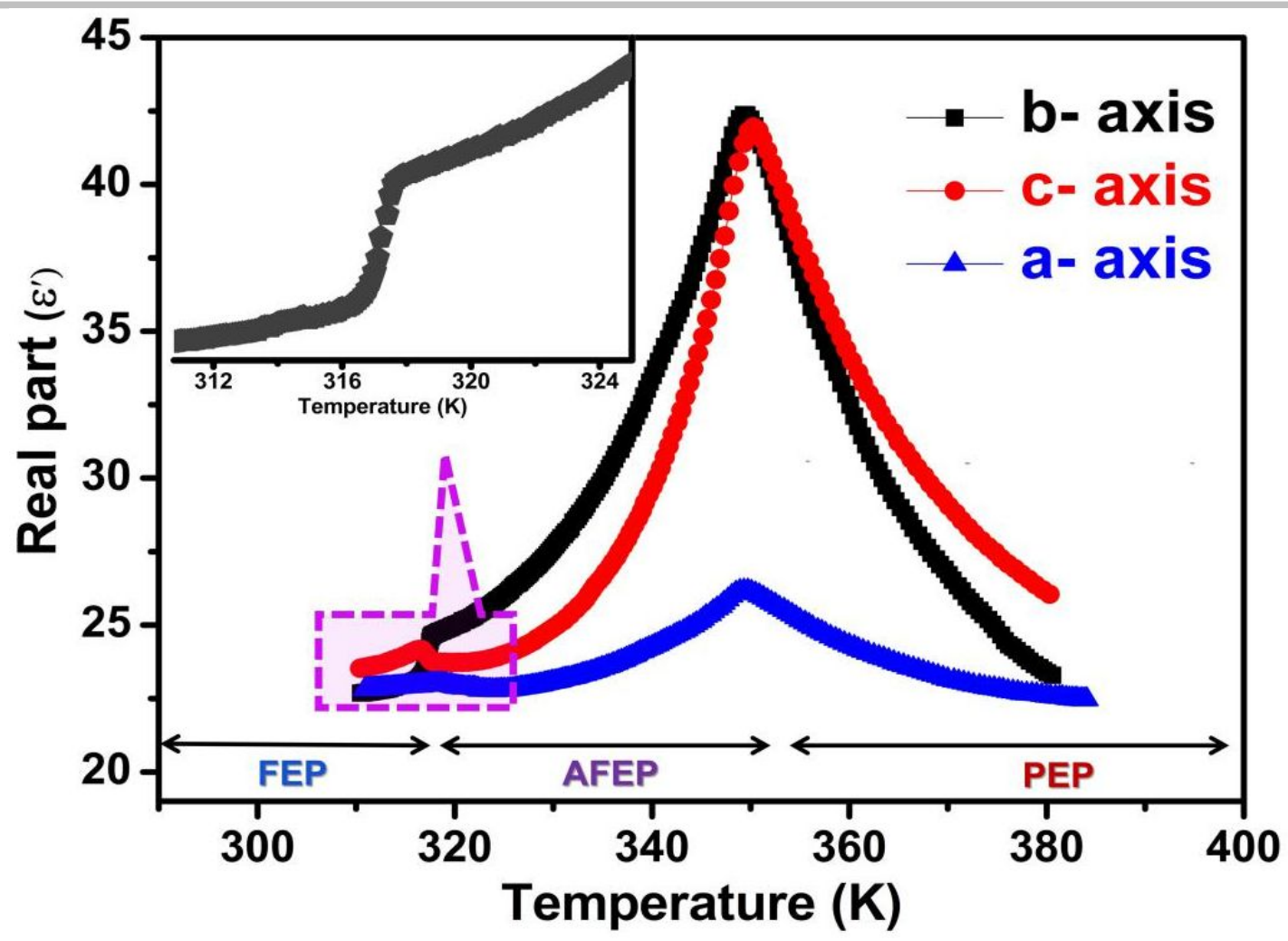

Figure S5. Temperature dependence of dielectric properties of 1. It is significant that the $\varepsilon^{\prime}$ values along the $b$ - and $c$ - axes exhibit distinctly peak-like dielectric anomalies around $T_{2}$, while quite a small anomaly is observed along the $a$ - axis. This result reveals its dielectric anisotropy.

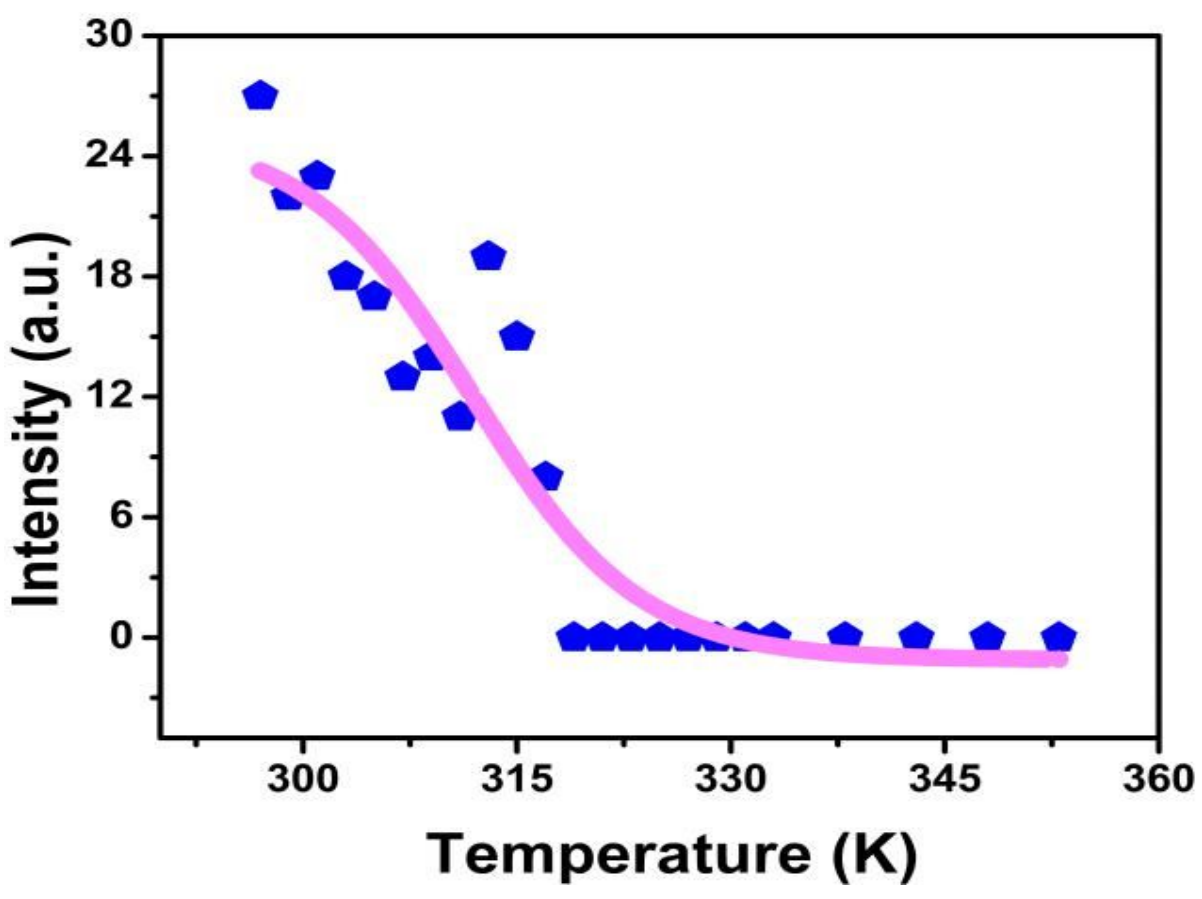

Figure S6. Variable-temperature SHG intensities of 1 collected at $670 \mathrm{~nm}$ in the temperature range of $293-365 \mathrm{~K}$. 


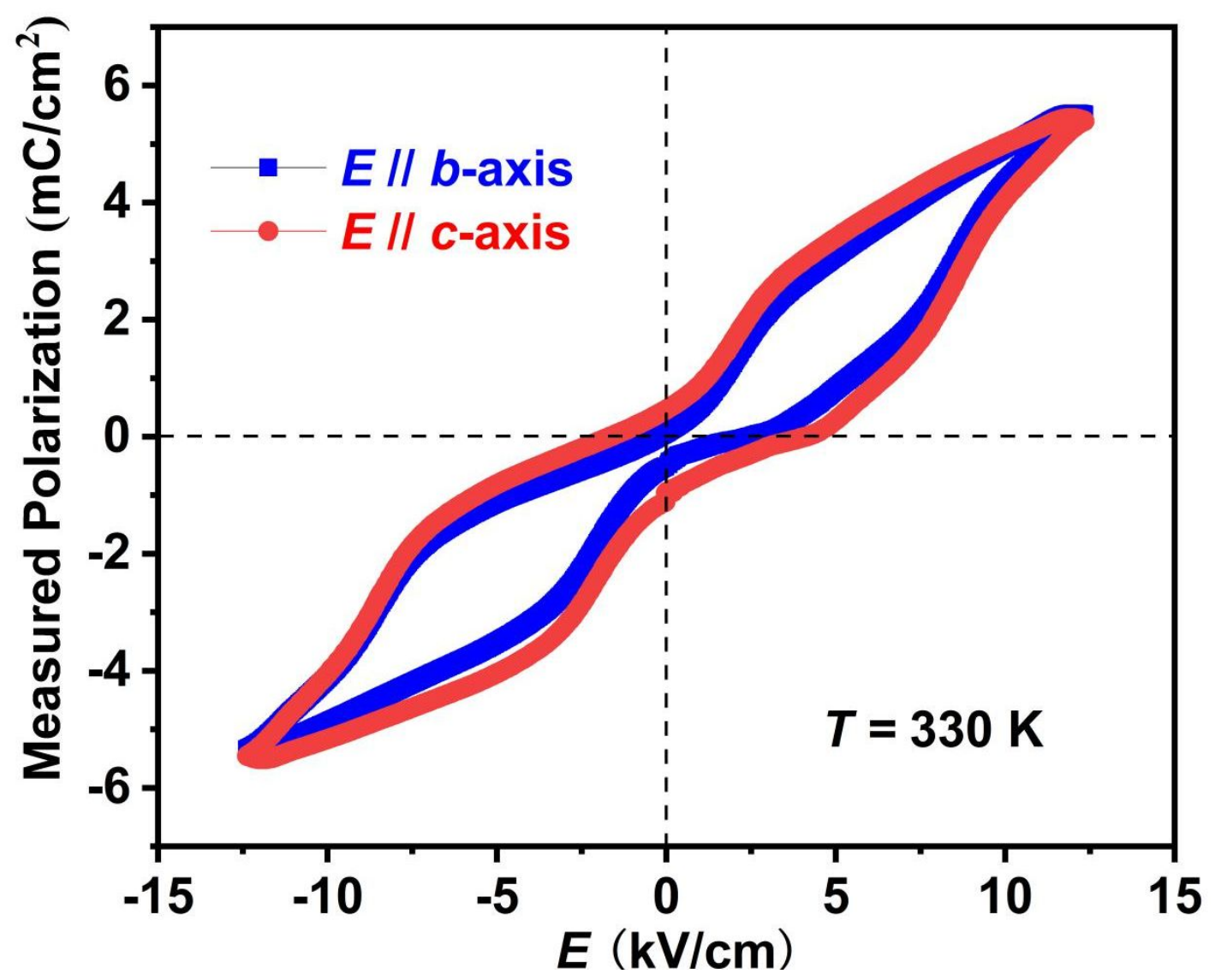

Figure S7. The double $P$ - $E$ hysteresis loops measured along $b$ - and $c$ - axes at $330 \mathrm{~K}$.

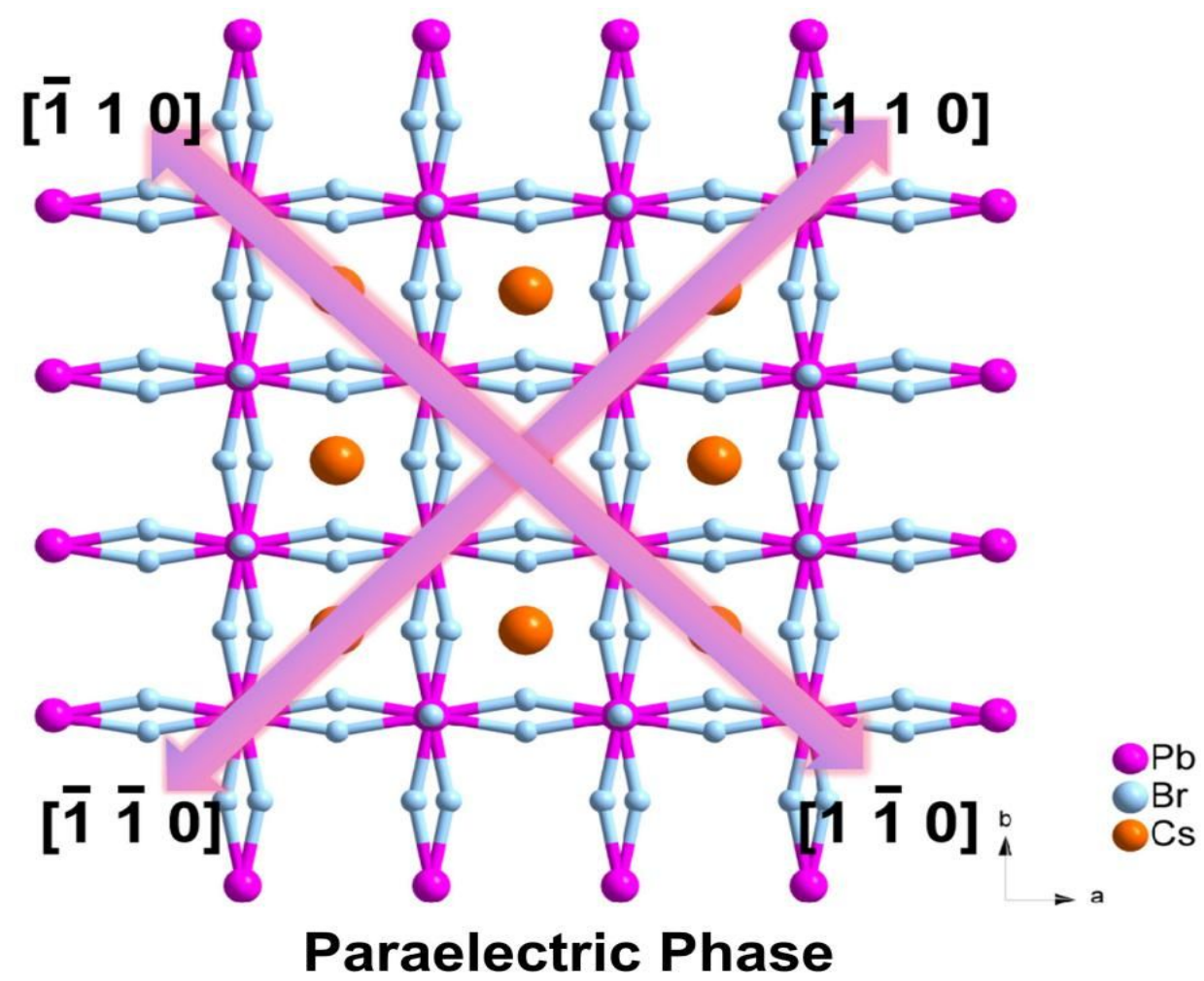

Figure S8. Diagram for the equivalent [110] directions of 1 at paraelectric phase, coinciding with the polar $c$-axis at ferroelectric phase. This result discloses the biaxial nature for ferroelectricity. 


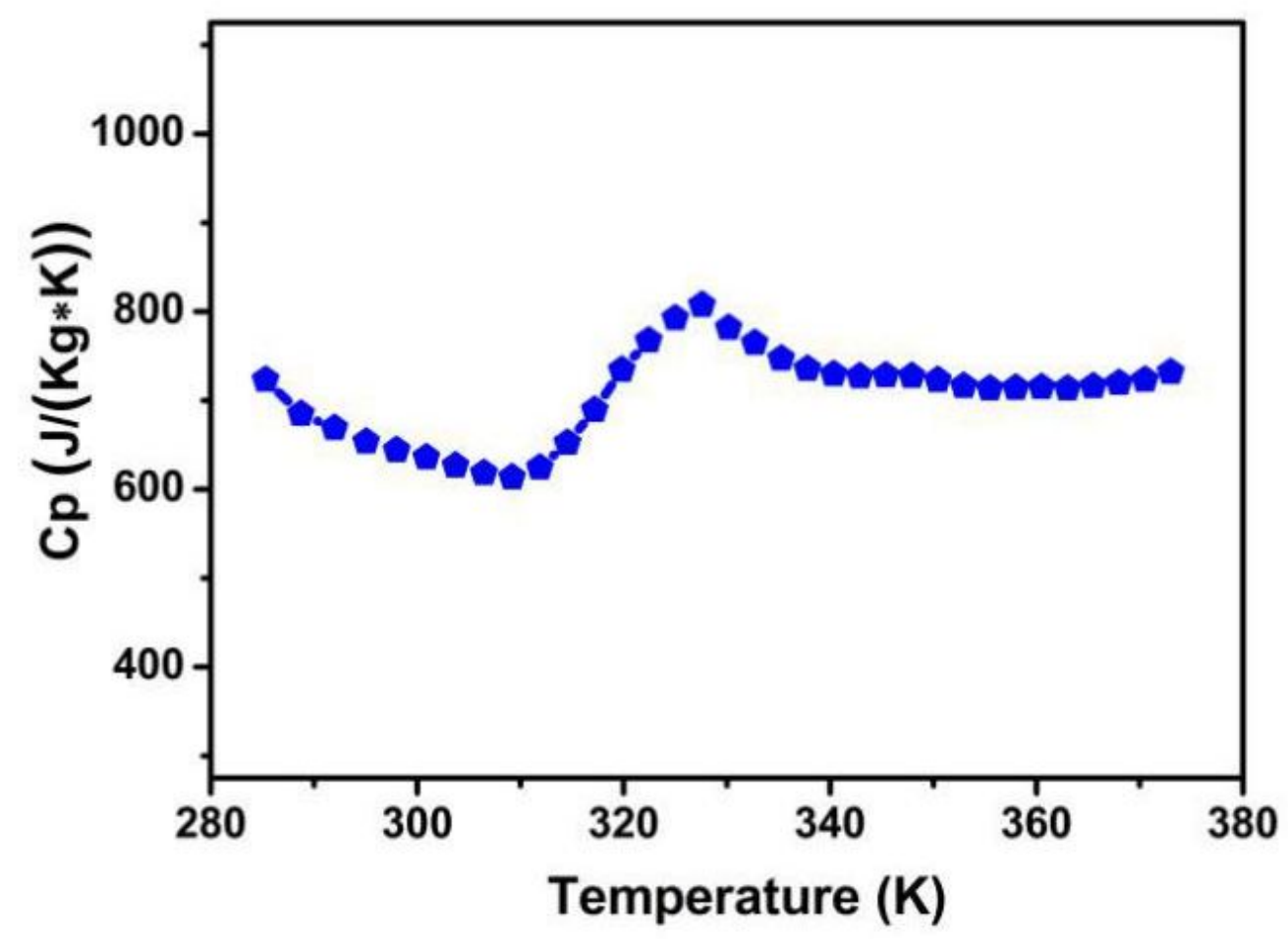

Figure S9. Specific heat $\left(C_{\mathrm{p}}\right)$ measured on crystal of $\mathbf{1}$ at different temperatures.
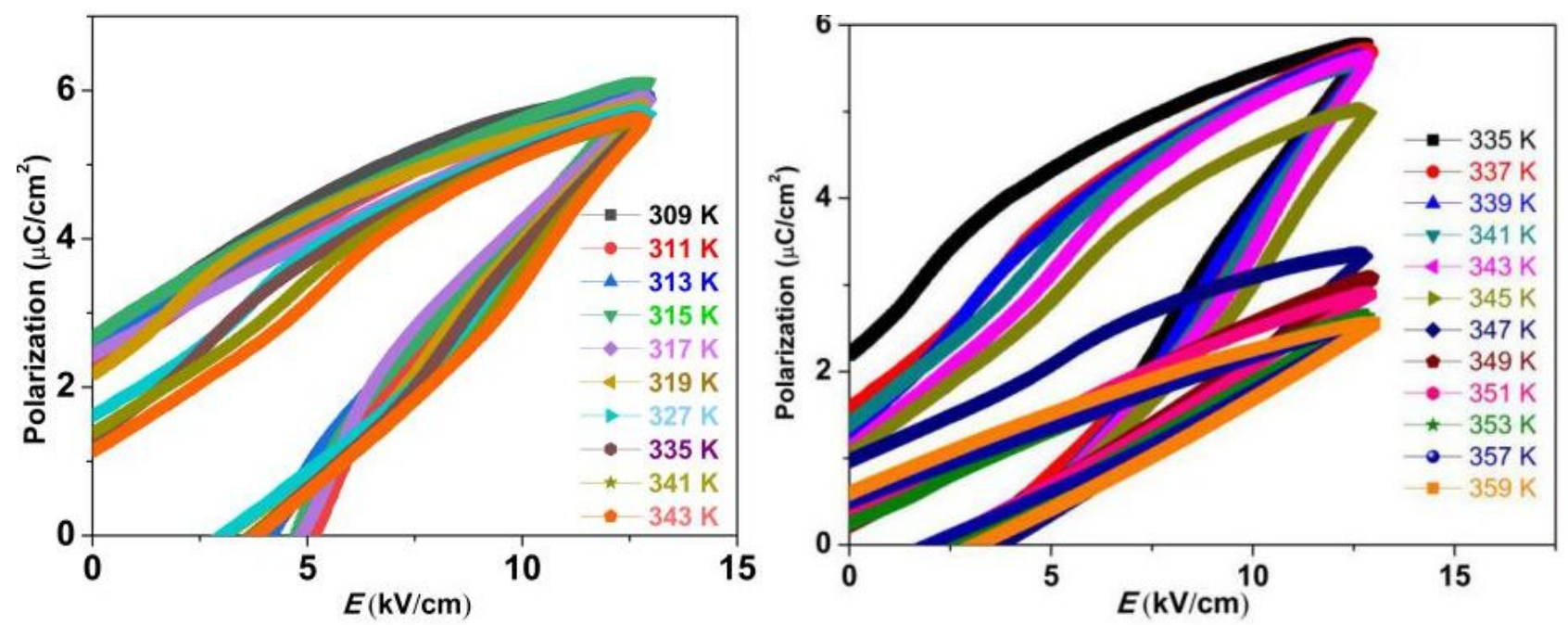

Figure S10. Half $P$ - $E$ loops as a function of temperature through the phase transitions. 


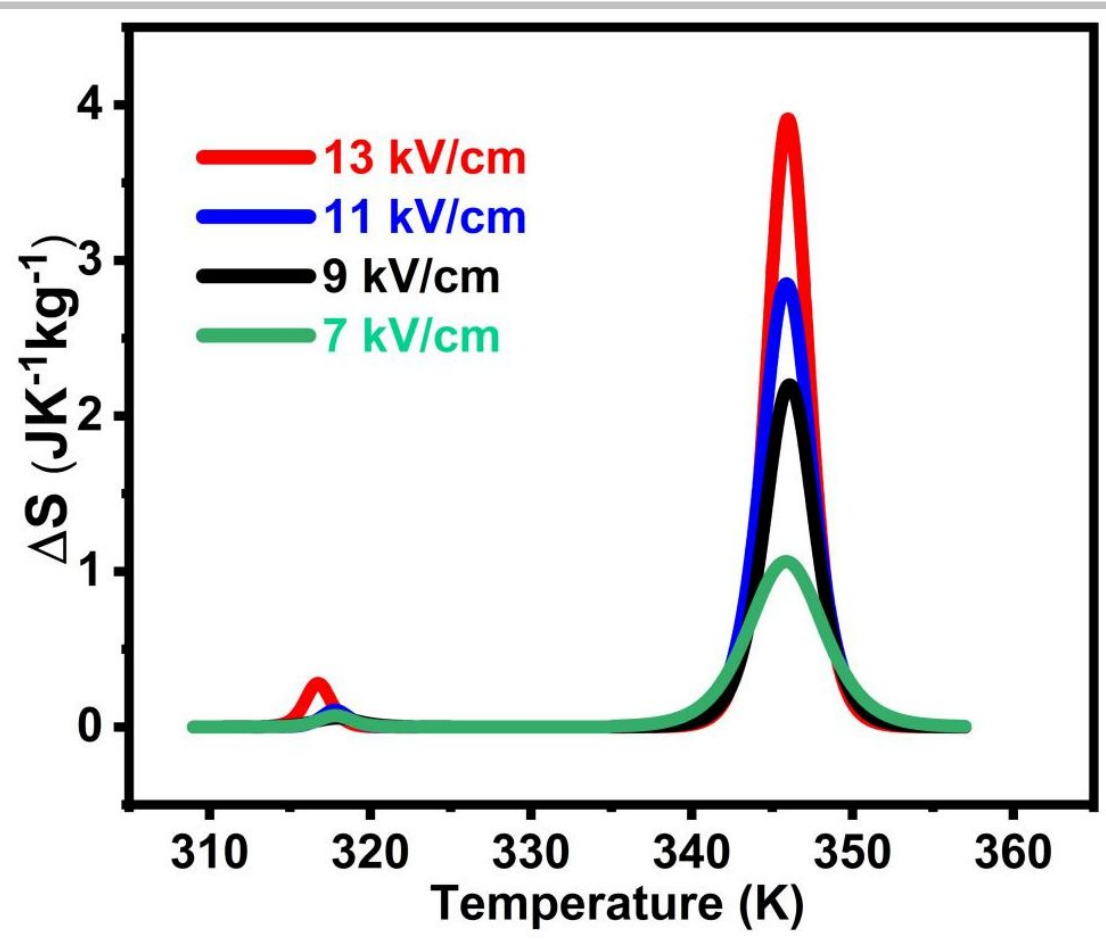

Figure S11. The EC-induced entropy changes $(\Delta S)$ under different external electric fields. 


\section{Tables}

Table S1. Crystal data and structure refinement for 1 at 310, 323 and $373 \mathrm{~K}$.

\begin{tabular}{|c|c|c|c|}
\hline Temperature & FEP (at $310 \mathrm{~K}$ ) & AFEP (at 323K) & PEP (at $373 \mathrm{~K}$ ) \\
\hline $\begin{array}{l}\text { Empirical formula } \\
\text { Crystallographic } \\
\text { system, Space group }\end{array}$ & $\begin{array}{r}\mathrm{C}_{10} \mathrm{H}_{28} \mathrm{Br}_{7} \mathrm{CsN}_{2} \mathrm{~Pb}_{2} \\
\text { Orthorhombic, } C m c 2_{1}\end{array}$ & $\begin{array}{c}\mathrm{C}_{10} \mathrm{H}_{28} \mathrm{Br}_{7} \mathrm{CsN}_{2} \mathrm{~Pb}_{2} \\
\text { Orthorhombic } \\
\mathrm{Pbcm}\end{array}$ & $\begin{array}{l}\mathrm{C}_{10} \mathrm{H}_{28} \mathrm{Br}_{7} \mathrm{CsN}_{2} \mathrm{~Pb}_{2} \\
\text { Tetragonal, I4/mmm }\end{array}$ \\
\hline \multirow{3}{*}{ Cell parameters } & $a=44.406 \AA(4)$ & $a=8.2371(3)$ & $a=5.8341 \AA(10)$ \\
\hline & $b=8.2275 \AA(7)$ & $b=8.2257(4)$ & $b=5.8341 \AA(10)$ \\
\hline & $c=8.2297 \AA(7)$ & $c=44.415(3)$ & $c=44.6405 \AA(2)$ \\
\hline $\mathrm{V}\left(\AA^{3}\right)$ & $3006.7(5)$ & 3009.3(3) & $1519.42(7)$ \\
\hline Z & 4 & 4 & 2 \\
\hline $\begin{array}{l}\text { Calculated density } \\
\left(\mathrm{g} / \mathrm{cm}^{3}\right)\end{array}$ & 2.834 & 2.832 & 2.804 \\
\hline$F(000)$ & 2264 & 2264 & 1132 \\
\hline \multirow[t]{2}{*}{ Theta range $\left({ }^{\circ}\right)$} & $5.036-51.222$ & $4.946-54.25$ & $7.044-55.036$ \\
\hline & $-53 \leq h \leq 53$ & $-10 \leq h \leq 10$ & $-7 \leq h \leq 6$ \\
\hline \multirow[t]{2}{*}{ Limiting indices } & $-9 \leq k \leq 10$ & $-10 \leq k \leq 10$ & $-7 \leq k \leq 7$ \\
\hline & $-10 \leq l \leq 9$ & $-57 \leq l \leq 55$ & $-57 \leq l \leq 52$ \\
\hline $\begin{array}{l}\text { Reflections collected / } \\
\text { unique }\end{array}$ & $\begin{array}{c}7321 / 2708 \\
{\left[R_{\mathrm{int}}=0.0533\right]}\end{array}$ & $\begin{array}{c}18936 / 3373 \\
{\left[R_{\mathrm{int}}=0.0564\right]}\end{array}$ & $\begin{array}{c}5509 / 602 \\
{\left[R_{\mathrm{int}}=0.0564\right]}\end{array}$ \\
\hline Completeness $\quad(\%)$ & 95 & 100 & 98.9 \\
\hline $\begin{array}{l}\text { Data/restraints/parameter } \\
\mathrm{s}\end{array}$ & $2708 / 43 / 107$ & $3373 / 81 / 106$ & $602 / 86 / 75$ \\
\hline GOF. & 1.028 & 1.045 & 1.055 \\
\hline $\begin{array}{l}\text { Final } \mathrm{R} \text { indices }[\mathrm{I}>2 \sigma \\
\text { (I) ] }\end{array}$ & $\begin{array}{l}R_{1}=0.0561 \\
w R_{2}=0.1056\end{array}$ & $\begin{array}{l}R_{1}=0.0458 \\
w R_{2}=0.1058\end{array}$ & $\begin{array}{l}R_{1}=0.0288 \\
w R_{2}=0.0638\end{array}$ \\
\hline $\mathrm{R}$ indices (all data) & $\begin{array}{l}R_{1}=0.1119 \\
w R_{2}=0.1253\end{array}$ & $\begin{array}{l}R_{1}=0.0867 \\
w R_{2}=0.1264\end{array}$ & $\begin{array}{l}R_{1}=0.0337 \\
w R_{2}=0.0668\end{array}$ \\
\hline
\end{tabular}


Table S2. Comparison of the electrocaloric properties among different materials.

\begin{tabular}{|c|c|c|c|c|c|c|c|}
\hline Material & Form & $T(\mathrm{~K})$ & $\Delta E(\mathrm{kV} / \mathrm{cm})$ & $\Delta S\left(\mathrm{JK}^{-1} \mathrm{~kg}^{-1}\right)$ & $\Delta T(\mathrm{~K})$ & $\Delta T / \Delta E\left(\mathrm{~K} \mathrm{~m} \mathrm{kV}^{-1}\right)$ & Ref. \\
\hline $\begin{array}{l}\mathrm{BaZrTiO}_{3}(\mathrm{X}=0.2) \text { (low } \\
\text { filed) }\end{array}$ & ceramic & 311 & 21 & 1.9 & 1.1 & 5.2 & 1 \\
\hline $\mathrm{BaZr}_{0.2} \mathrm{Ti}_{0.8} \mathrm{O}_{3}$ & thick film & 313 & 97 & 8.5 & 4.9 & 5.0 & 2 \\
\hline $\mathrm{BaTiO}_{3}$ & ceramic & 391 & 7.5 & - & 0.4 & 5.3 & 3 \\
\hline $\mathrm{BaTiO}_{3}$ & MLCC & 353 & 800 & 10.1 & 7.1 & 0.88 & 4 \\
\hline $\mathrm{BaTiO}_{3}$ & $\begin{array}{l}\text { single } \\
\text { crystal }\end{array}$ & 402 & 12 & 2.2 & 0.9 & 7.5 & 5 \\
\hline $\mathrm{BaTiO}_{3}$ & $\begin{array}{l}\text { single } \\
\text { crystal }\end{array}$ & 413 & 10 & - & 1.6 & 16 & 6 \\
\hline $\begin{array}{c}\mathrm{Pb}\left(\mathrm{Mg}_{1 / 3} \mathrm{Nb}_{2 / 3}\right)_{0.7} 5 \mathrm{Ti}_{0.25} \mathrm{O} \\
3\end{array}$ & $\begin{array}{l}\text { single } \\
\text { crystal }\end{array}$ & 383 & 25 & - & 1.1 & 4.4 & 7 \\
\hline $\mathrm{Sr}_{0.75} \mathrm{Ba}_{0.25} \mathrm{Nb}_{2} \mathrm{O}_{6}$ & $\begin{array}{l}\text { single } \\
\text { crystal }\end{array}$ & 353 & 10 & - & 0.4 & 4 & 8 \\
\hline BNBT/BCZT (F) & bilayer & 370 & 620 & 26.1 & 23 & 3.7 & 9 \\
\hline $\mathrm{BaHf}_{\mathrm{x}} \mathrm{Ti}_{1-\mathrm{x}} \mathrm{O}_{3}(\mathrm{x}=0.03)$ & Ceramic & 343 & 10 & - & 0.35 & 3.5 & 10 \\
\hline $\begin{array}{l}\text { P(VDF-TrFE-CFE-CTFE) } \\
56.1 / 33.2 / 5.9 / 4.8 \mathrm{~mol} \%\end{array}$ & Polymer & 308 & $10^{3}$ & 40 & 8 & $8 \times 10^{-1}$ & 11 \\
\hline $\begin{array}{l}\text { P(VDF-TrFE) } \\
55 / 45 \mathrm{~mol} \mathrm{\%}\end{array}$ & Polymer & 300 & $2.09 \times 10^{3}$ & 62 & 12.6 & $6 \times 10^{-1}$ & 12 \\
\hline $5 \mathrm{CB}$ & Fluidic & 312 & 900 & 90 & 24 & 2.6 & 13 \\
\hline This Work & $\begin{array}{l}\text { single } \\
\text { crystal }\end{array}$ & 350 & 13 & 4.1 & 2.0 & 15.4 & $\begin{array}{l}\text { This } \\
\text { Work }\end{array}$ \\
\hline
\end{tabular}




\section{Reference}

[1] Qian, X.-S.; Ye, H.-J.; Zhang, Y.-T.; Gu, H.; Li, X.; Randall, C. A.; Zhang, Q. M. Giant electrocaloric response over a broad temperature range in modified $\mathrm{BaTiO}_{3}$ ceramics. Adv. Funct. Mater., 2014, 24, 1300-1305.

[2] Ye, H.-J.; Qian, X.-S.; Jeong, D.-Y.; Zhang, S.; Zhou, Y.; Shao, W.-Z.; Zhen, L.; Zhang, Q. M. Giant electrocaloric effect in $\mathrm{BaZr}_{0.2} \mathrm{Ti}_{0.8} \mathrm{O}_{3}$ thick film. Appl. Phys. Lett. 2014, 105, 152908.

[3] Karchevskii, A. Electrocaloric effect in polycrystalline barium titanate. Sov. Phys. Solid State, 1962, 3, $2249-2254$.

[4] Bai, Y.; Zheng, G.-P.; Ding, K.; Qiao. L.; Shi, S.-Q.; Guo, D. The giant electrocaloric effect and high effective cooling power near room temperature for $\mathrm{BaTiO}_{3}$ thick film. J. Appl. Phys. 2011, 110, 094103.

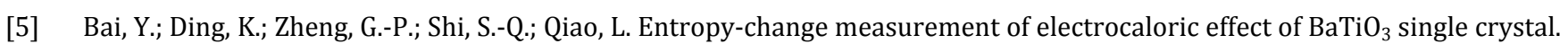
Phys. Status Solidi A, 2012, 209, 941-944.

[6] Moya, X.; Stern-Taulats, E.; Crossley, S.; González-Alonso, D.; Kar-Narayan, S.; Planes, A.; Mañosa, L.; Mathur, N. D. Giant electrocaloric strength in single-crystal $\mathrm{BaTiO}_{3}$. Adv. Mater. 2013, 25, 1360-1365.

[7] Sebald, G.; Seveyrat, L.; Guyomar, D.; Lebrun, L.; Guiffard, B.; Pruvost, S. Electrocaloric pyroelectric properties of $0.75 \mathrm{~Pb}\left(\mathrm{Mg}_{1 / 3} \mathrm{Nb}_{2 / 3}\right) \mathrm{O}_{3}-0.25 \mathrm{PbTiO}_{3}$ single crystals. J. Appl. Phys. 2006, 100, 124112.

[8] Goupil, F. L.; Axelsson, A.-K.; Dunne, L. J.; Valant, M.; Manos, G.; Lukasiewicz, T.; Dec, J.; Berenov, A.; Alford, N. M. Anisotropy of the electrocaloric effect in lead free relaxor ferroelectrics. Adv. Energy Mater. 2014, 4, 1301688.

[9] Shirsath, S. E.; Cazorla, C.; Lu, T.; Zhang, L.; Yay, Y. Y.; Lou, X.; Liu, Y.; Li, S.; Wang, D. Interface-charge induced giant electrocaloric effect in lead-free ferroelectric thin-film bilayers. Nano Lett. 2020, 20, 1262-1271.

[10] Li, J.; Zhang, D.; Qin, S.; Li, T.; Wu, M.; Wang, D.; Bai, Y.; Lou, X. Large room-temperature electrocaloric effect in lead-free $\mathrm{BaHf}_{\mathrm{x}} \mathrm{Ti}_{1-\mathrm{x}} \mathrm{O}_{3}$ ceramics under low electric field. Acta Mater., 2016, 115, 58-67.

[11] Chen, X.; Xu, W.; Lu, B.; Wang, Q.; Zhang, Q. M. Towards electrocaloric heat pump-A relaxor ferroelectric polymer exhibiting large electrocaloric response at low electric field. Appl. Phys. Lett. 2018, 113, 113902.

[12] Neese, B.; Chu, B.; Lu, S.-G.; Wang, Y.; Furman, E.; Zhang, Q. M. Large Electrocaloric Effect in Ferroelectric Polymers Near Room Temperature. Science 2008, 321, 821-823.

[13] Qian, X.-S.; Lu, S.-G.; Li, X.; Gu, H.; Chien, L.-C.; Zhang, Q. Large Electrocaloric effect in a dielectric liquid possessing a large dielectric anisotropy near the isotropic-nematic transition. Adv. Funct. Mater. 2013. 23,2894-2898. 